\section{JAHRESTAGUNG DER AMERICAN HEART ASSOCIATION}

\section{(.}

\section{Reichlich neue Erkenntnisse rund ums Herz}

Wie jedes Jahr im November trafen sich Herzspezialisten vergangene Woche zur Jahrestagung der American Heart Association in Orlando, einem der größten Medizin-Kongresse weltweit. Die wichtigsten Studien, über die wir in der nächsten MMW ausführlicher berichten werden, tragen folgende Schlagzeilen:

- Prognose verbessert bei akutem Koronarsyndrom: Rivaroxaban senkt Risiken für Tod, Herzinfarkt und Schlaganfall (ATLASACS 2 TIMI 52-Studie)

- Dronedaron kontraindiziert bei permanentem Vorhofflimmern: Verdopplung des Risikos für Herzschwäche, Schlaganfall und Tod (PALLAS-Studie)

- Rückschlag für die HDL-Anhebung: Retardiertes Niacin bei stabilen KHK-Patienten

\section{SOFTDRINKS MIT ZUCKERZUSATZ}

\section{Zwei pro Tag sind zu viel für Herz und Figur}

Frauen, aufgepasst: Cola, Fanta und ähnliche Softdrinks mit Zuckerzusatz ruinieren die Figur und erhöhen das Risiko für Herzerkrankungen und Diabetes. Zumindest dann, wenn zwei oder mehr pro Tag getrunken werden. Forscher fanden heraus, dass langfristig die Triglyzeride ansteigen und die Glukosetoleranz abnimmt. Außerdem legen die Damen an den Hüften zu, ohne zwangsläufig an Gewicht zuzunehmen. Dergleichen wurde bei Männern nicht beobachtet.

Abstract 8438 mit gut kontrolliertem LDL ohne Wirkung (AIM-HIGH-Studie)

- Langfristig Gewicht reduziert: Erfolgreiche Verhaltensänderung durch Patientenmotivation per Telefon und E-Mail (POWER-Studie)

- Gute Nachricht für Patienten mit proximaler PAVK: Training wirksamer als Stenting (CLEVER-Studie)

- Sparen an der falschen Stelle: Medikamenten-Zuzahlung bei Herzmedikamenten erhöht Komplikationsrate (Post-MI FREEE-Studie).

Abstracts zu den nachfolgenden Meldungen unter: circ.ahajournals. org/content/vol124/21_Meeting Abstracts. Oder scannen Sie den QR-Code mit Ihrem Smartphone oder Tablet-PC.

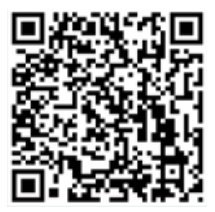

\section{PATIENT MIT HERZSCHWÄCHE}

\section{Schlechtere Prognose bei Vitamin-C-Defizit}

Patienten mit Herzinsuffizienz sollten ausreichend Obst und Gemüse verzehren, um ein Vitamin-C-Defizit zu vermeiden. Denn erstmals wurde in einer Studie festgestellt, dass eine ausreichende Vitamin-C-Aufnahme mit besseren Überlebensaussichten assoziiert ist. Patienten mit niedrigen Vitamin-C-Spiegeln und konsekutiv erhöhten Werten für hochsensitives CRP (über 3 $\mathrm{mg} / \mathrm{l}$ ) hatten eine doppelt so hohe Mortalität in der kleinen Studie, die 212 Patienten über ein Jahr beobachtete. $\quad$ Abstract 14667

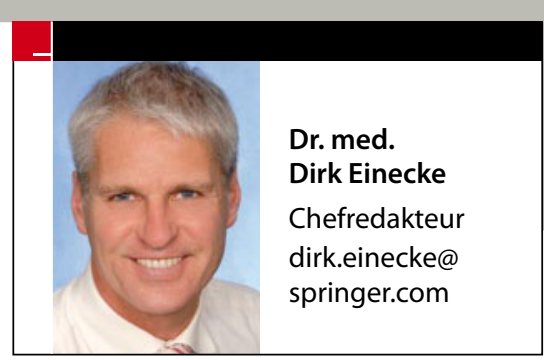

PROPHYLAXE BEIM ZAHNARZT

\section{Zahnsteinentfernung schützt Herz und Hirn}

Wer sich wenigstens einmal jährlich einer professionellen Dentalhygiene unterzieht, hat ein um $24 \%$ geringes Herzinfarktrisiko sowie ein um 13\% geringeres Schlaganfallrisiko als Personen, die solche Dienste nie in Anspruch nehmen. Dies folgt aus einer Studie aus Taiwan mit über 100000 gesunden Probanden, die sieben Jahre lang nachverfolgt wurden. Allerdings waren in der Studie die Risikofaktoren der Probanden für Herzinfarkt und Schlaganfall nicht bekannt und entsprechend konnte nicht adjustiert werden.

Abstract 17704

\section{BLUTDRUCK SENKEN}

\section{Drei Kiwis wirksamer als ein Apfel}

Schon der berühmte „apple a day" hält dem Vernehmen nach den "doctor away“. Noch segensreicher scheint der Verzehr von drei Kiwis am Tag zu sein, zumindest was die Blutdrucksenkung betrifft: $3,6 \mathrm{mmHg} z u-$ gunsten der Kiwis im Vergleich zum Apfel, so lautete das Ergebnis des Obstduells über acht Wochen bei 130 Hypertonikern. Das Therapieergebnis war mit 24-Std.-RR-Messungen bestimmt worden. Kiwis enthalten Lutein, das antioxidativ wirkt.

Abstract 14035

\title{
Fit mit 50, gesünder mit 65
}

Ein Schlüssel zum gesunden Altern ist offenbar die Fitness im mittleren Alter. Wem das gelingt, der reduziert sein Risiko für chronische Erkrankungen im Alter von 65 Jahren signifikant: Frauen um 36\% und
Männer um 39\%. Die Erkenntnis folgt aus einer Studie mit knapp 19000 Personen im Alter von 49 Jahren, die langfristig beobachtet wurden und deren Fitnessgrad bekannt war.
Abstract 15850

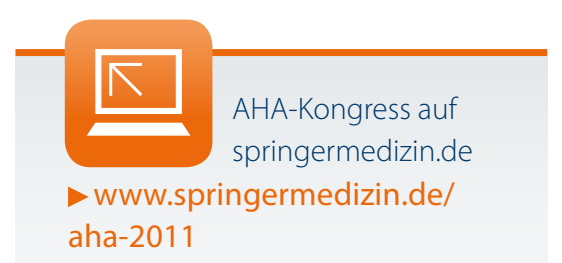

